\title{
Voluntary Control of Epileptiform Spike-Wave Discharges in Awake Rats
}

\author{
DJeremy A. Taylor, ${ }^{1}$ Krista M. Rodgers, ${ }^{1}$ Florencia M. Bercum, ${ }^{1}$ CCarmen J. Booth, ${ }^{2}$ F. Edward Dudek, ${ }^{3}$ \\ and ${ }^{\circ D}$ Daniel S. Barth ${ }^{1}$ \\ ${ }^{1}$ Department of Psychology and Neuroscience, University of Colorado, Boulder, Colorado 80309, 2Department of Comparative Medicine, Yale University \\ School of Medicine, New Haven, Connecticut 06520, and '3Department of Neurosurgery, University of Utah School of Medicine, Salt Lake City, Utah 84108
}

Genetically inherited absence epilepsy in humans is typically characterized by brief (seconds) spontaneous seizures, which involve spike-wave discharges (SWDs) in the EEG and interruption of consciousness and ongoing behavior. Genetic (inbred) models of this disorder in rats have been used to examine mechanisms, comorbidities, and antiabsence drugs. SWDs have also been proposed as models of complex partial seizures (CPSs) following traumatic brain injury (post-traumatic epilepsy). However, the ictal characteristics of these rat models, including SWDs and associated immobility, are also prevalent in healthy outbred laboratory rats. We therefore hypothesized that SWDs are not always associated with classically defined absence seizures or CPSs. To test this hypothesis, we used operant conditioning in male rats to determine whether outbred strains, Sprague Dawley and Long-Evans, and/or the inbred WAG/Rij strain (a rat model of heritable human absence epilepsy) could exercise voluntary control over these epileptiform events. We discovered that both inbred and outbred rats could shorten the duration of SWDs to obtain a reward. These results indicate that SWD and associated immobility in rats may not reflect the obvious cognitive/behavioral interruption classically associated with absence seizures or CPSs in humans. One interpretation of these results is that human absence seizures and perhaps CPSs could permit a far greater degree of cognitive capacity than often assumed and might be brought under voluntary control in some cases. However, these results also suggest that SWDs and associated immobility may be nonepileptic in healthy outbred rats and reflect instead voluntary rodent behavior unrelated to genetic manipulation or to brain trauma.

Key words: absence; epilepsy; SWD

\section{Significance Statement}

Our evidence that inbred and outbred rats learn to control the duration of spike-wave discharges (SWDs) suggests a voluntary behavior with maintenance of consciousness. If SWDs model mild absence seizures and/or complex partial seizures in humans, then an opportunity may exist for operant control complementing or in some cases replacing medication. Their equal occurrence in outbred rats also implies a major potential confound for behavioral neuroscience experiments, at least in adult rats where SWDs are prevalent. Alternatively, the presence and voluntary control of SWDs in healthy outbred rats could indicate that these phenomena do not always model heritable absence epilepsy or post-traumatic epilepsy in humans, and may instead reflect typical rodent behavior.

\section{Introduction}

Synchronous 7-12 Hz spike-wave discharges (SWDs) are commonly detected in electrocortical recordings of rats during awake

Received Oct. 19, 2016; revised May 7, 2017; accepted May 9, 2017.

Author contributions: J.A.T., K.M.R., F.E.D., and D.S.B. designed research; J.A.T., K.M.R., F.M.B., and D.S.B. performed research; C.J.B. contributed unpublished reagents/analytic tools; J.A.T. and D.S.B. analyzed data; J.A.T., F.E.D., and D.S.B. wrote the paper.

This work was supported by the U.S. Army Medical Research and Material Command (Grants PR100040 and EP150033) and by Citizens United for Research in Epilepsy (Grant \#7153).

F.E. Dudek has received consulting fees from Rugen Biomedical and Neurona Therapeutics. He has equity interest in and receives consultant fees from Epitel, a company that makes telemetric recording devices; however, this work did not use these devices. The other authors declare no competing financial interests. immobility. SWDs have a rapid onset and termination, are brief (seconds), are typically accompanied by vibrissa twitching, and are usually followed by rapid resumption of previous behavior and electrical activity. The transient and paroxysmal appearance of SWDs with associated immobility and rhythmic facial movements has led to the belief that SWDs represent a hallmark of seizures in both inbred genetic (i.e., absence) and acquired (posttraumatic) models of epilepsy. If SWDs and associated immobil-

Correspondence should be addressed to Daniel S. Barth, Department of Psychology and Neuroscience, University of Colorado, Campus Box 345, Boulder, C0 80309. E-mail: daniel.barth@colorado.edu.

DOI:10.1523/JNEUROSCI.3235-16.2017

Copyright $\odot 2017$ the authors $\quad 0270-6474 / 17 / 375861-09 \$ 15.00 / 0$ 
ity are consistently epileptic, it is of concern because frequent SWDs (many per hour) are also observed in many healthy adult outbred strains of laboratory rats used in behavioral neuroscience research and preclinical studies of epilepsy (Vergnes et al., 1982; Kaplan, 1985; Buzsaki et al., 1990; Kelly, 2004; Shaw, 2004; Pearce et al., 2014) where unrecognized seizures could present a significant confound. However, the role of SWDs in rat epilepsy is a matter of continuing debate (Kaplan, 1985; Wiest and Nicolelis, 2003; Kelly, 2004; Shaw, 2004; D'Ambrosio and Miller, 2010; Dudek and Bertram, 2010; Rodgers et al., 2015).

SWDs and associated immobility in a long-studied rat model of traumatic brain injury based on fluid percussion injury (FPI) have been proposed to reflect nonconvulsive, complex partial (i.e., focal dyscognitive; Berg et al., 2010) seizures (CPSs) of human post-traumatic epilepsy (PTE; D'Ambrosio et al., 2004, 2009). SWDs appear early after FPI, with increasing prevalence over months, thus leading to the interpretation that SWDs in the FPI model are equivalent to CPSs during the progressive development of human PTE. Similar SWDs and associated immobility—proposed to be equivalent to CPSs - have also been reported in rat models of other injury-induced epilepsies, specifically neonatal hypoxia (Rakhade et al., 2011) and a hyperthermia-induced convulsions model of febrile seizures in immature rats (Dubé et al., 2006). Thus, at least three animal models of acquired epilepsy are based on events similar to the SWDs of inbred models of absence epilepsy as well as to the SWDs of healthy outbred rats.

Whether SWDs reflect PTE has been challenged by evidence of an equal prevalence in age-matched uninjured rats (Pearce et al., 2014; Rodgers et al., 2015). We recently reported that epochs of SWDs and associated immobility are indistinguishable between FPI and sham-injured rats (Rodgers et al., 2015). We concluded that since their occurrence did not require prior injury, they must reflect heritable seizures typically reported in rats inbred for SWDs over multiple generations and used as genetic models of absence epilepsy for decades. Three hallmarks characterize typical human absence (petit mal) seizures: (1) a distinct heritability or clear genetic component, (2) a clinical manifestation of sudden and brief but profound impairment of consciousness with behavioral arrest (i.e., absence), and (3) a concomitant appearance of classic, large-amplitude, $3 \mathrm{~Hz}$, paroxysmal SWDs in the EEG. While established inbred rodent models of human absence epilepsy approximate these hallmarks, our observations of SWDs were in outbred Sprague Dawley rats (SD rats; Rodgers et al., 2015). Episodes of SWDs and associated immobility reported in most outbred strains suggest that rats used in behavioral and preclinical studies have frequent seizures that could seriously confound results of many types of studies, particularly if undetected. Alternatively, SWDs in healthy outbred rats may not represent epileptic seizures. We hypothesized that SWDs in healthy outbred laboratory rats are not seizures associated with behavioral arrest; rather, they are a voluntary behavior of many strains of laboratory rats. Since both complex partial (focal dyscognitive) and absence seizures in humans are spontaneous (involuntary) and, by definition, typically associated with profound impairment of cognition, a central prediction of our hypothesis is that rats remain conscious during SWDs and can control SWDs. Here we report the first test of this prediction using a simple operant learning paradigm in outbred SD and Long-Evans (LE) rats, the most common strains used in behavioral neuroscience. For comparison, we used the same paradigm with $W A G / R i j$ rats inbred to model absence seizures in humans.

\section{Materials and Methods}

Healthy adult ( $8-10$-month-old) male SD and LE rats $(n=5$ per group; Envigo) and inbred WAG/RijCmcr (WAG/Rij) rats (6-10-month-old; $n=5$; Department of Comparative Medicine, Yale University School of Medicine, New Haven, CT) were studied. Older rats were used because they typically display more SWDs than younger rats (Rodgers et al., 2015). Rats were housed under standard laboratory conditions in a temperature-controlled and relative humidity-controlled (i.e., $20 \pm 1^{\circ} \mathrm{C}$ and $60 \%$, respectively) environment with a normal $12 \mathrm{~h}$ light/dark cycle (lights on 7:00 A.M. to 7:00 P.M.). Food and water were available ad libitum. All procedures were performed in accordance with University of Colorado Institutional Animal Care and Use Committee guidelines for the humane use of laboratory rats in biological research.

\section{Electrocorticogram recording}

Epidural bilateral stainless steel screw electrodes were implanted using aseptic surgical procedures. Two electrode screws were placed bilaterally over the approximate orofacial region of somatosensory cortex [anteroposterior (AP), $-2.0 \mathrm{~mm}$; mediolateral $(\mathrm{ML}), 4.0 \mathrm{~mm}$ ], one reference electrode screw (AP, $3.0 \mathrm{~mm}$; ML, $1.0 \mathrm{~mm}$ ), one ground electrode screw (AP, $-7.5 \mathrm{~mm}$; ML, $1.0 \mathrm{~mm}$ ), and two anchor screws (AP, $4.0 \mathrm{~mm}$; ML, $2.0 \mathrm{~mm}$; AP, $-3 \mathrm{~mm}$; ML, $4 \mathrm{~mm}$, respectively) under isoflurane $(2.5 \%)$ anesthesia. During operant conditioning periods, animals were tethered to an electrode harness (Plastics One, 363) and slip-ring commutator (Plastics One, SL6C) permitting free movement for electrocorticogram (ECoG) monitoring throughout the experiment. ECoG signals were amplified $(10,000 \times)$, analog filtered $(1-200 \mathrm{~Hz}$ bandpass $)$, and digitized at $500 \mathrm{~Hz}$.

\section{Supervised pattern recognition of SWD}

In a manner similar to that of our previous studies (Rodgers et al., 2015), automated detection was used to quantify SWD events and to trigger a sucrose pellet dispenser for reward. ECoG data were analyzed in $1 \mathrm{~s}$ segments. Approximately 20 segments containing SWDs were visually selected to establish a template for each rat. Auto-covariance functions were computed for identified segments to capture the amplitude, frequency, and waveform morphology of the SWDs. Auto-covariance functions for SWD segments and similar functions for segments containing representative noise were used to train a support vector machine (SVM; Nandan et al., 2010; Orrù et al., 2012) to automatically discriminate between SWDs and noise. The trained SVM model for a given rat was then used to detect SWDs in that animal in real time for the remainder of the experiment.

\section{Power spectrum modeling}

To compare the spectral frequency distribution of SWDs between rats, power spectra of $1 \mathrm{~s}$ samples identified with SVM were computed using a Welch transform and averaged across $\sim 1000 \mathrm{~s}$ for a given rat. Power spectra were then averaged across all rats within each strain resulting in three averaged spectra. Similarities in the spectra were computed as the Pearson's product-moment correlation between all pairs.

\section{Experimental procedures}

After $2 \mathrm{~d}$ habituation to the recording cage ( $1 \mathrm{~h}$ per session), rats were initially trained over five daily sessions to receive unconditional rewards to associate the pellet tube with reward delivery. This was followed by 10 daily training sessions lasting $2 \mathrm{~h}$ each. The first five sessions began with $30 \mathrm{~min}$ no-reward recording followed by $30 \mathrm{~min}$ where a delayed ( $3 \mathrm{~s}$ ) reward was delivered after completing each SWD burst in the presence of continuous white noise as a contextual cue for reward versus no-reward periods. No-reward/reward periods were again presented to complete the session in a repeated-measures design. To counter-balance possible ordering effects, an additional five sessions were run in the same way but beginning with the reward instead of no-reward period. An SWD burst was classified as $\geq 3$ consecutive seconds of detected SWDs. After completing an SWD burst lasting $\geq 3 \mathrm{~s}$, non-SWD segments were monitored and a reward (single sucrose pellet) delivered only at the completion of $3 \mathrm{~s}$ without SWD. If the rat resumed SWD in $<3 \mathrm{~s}$, no reward was delivered 
and a new SWD burst began logging. An infrared beam interrupter was positioned at the tip of the pellet dispenser tube to record when the rats explored for reward. All segments of ECoG were stored on disk along with the output of the beam interrupter and event flags indicating SWD segments, SWD burst completions, and reward/no-reward deliveries. Periods of immobility were visually determined throughout the experiment. At the end of behavioral testing, rats were run through the conditioning procedure three additional times: once while under the effects of ethosuximide, once $24 \mathrm{~h}$ after ethosuximide administration, and once with saline. Ethosuximide (100 mg/kg; Sigma-Aldrich) was dissolved in saline $(0.9 \% \mathrm{NaCl})$ and injected intraperitoneally before initiating the $2 \mathrm{~h}$ conditioning procedure (saline injections followed the same procedure). Saline and ethosuximide injections were counterbalanced.

\section{Statistical analysis}

The durations and numbers of completed SWD bursts ( $\geq 3 \mathrm{~s}$ ) were quantified with four conditions (no-reward 1, reward 1, no-reward 2, reward 2) repeated over the 10 experimental days. Results are expressed as mean \pm SEM. A two-way, repeated-measures ANOVA was used to determine significant within-subject's effects as a function of condition. Post hoc comparisons were conducted to follow-up significant overall ANOVAs. Data were analyzed using SPSS Statistics software and, in all cases, statistical significance was set at $p \leq 0.05$.

\section{Terminology}

To avoid confusion and debate, we provide the following precise definitions for certain terms used in this paper: voluntary: done by choice, self-determined, unconstrained by interference (e.g., by spontaneous seizure); spontaneous: occurring without apparent external influence; seizure: a sudden attack, typically but not always associated with convulsions, sensory disturbances, and/or loss of consciousness; consciousness: the state of being aware; awareness: showing realization, perception or knowledge (e.g., of SWDs or behavior accompanying SWDs); cognition: the activities of thinking, understanding, learning, and remembering (e.g., learning the association between a reward period cue, such as white noise, termination of an SWD burst, and delayed reward); absence: inattention to the present surrounding (e.g., presence of reward cue) or occurrences (e.g., end of SWD burst).

\section{Results}

\section{Outbred SD rats}

A variety of sensory stimuli have been shown to terminate SWDs in outbred rats (Robinson and Gilmore, 1980; Semba et al., 1980; Vergnes et al., 1982; Buzsáki et al., 1990a; Wiest and Nicolelis, 2003; Shaw, 2004; Pearce et al., 2014; Rodgers et al., 2015). Figure $1 A$ depicts a typical computer-detected SWD burst (red) in an SD rat, where a reward was automatically administered after $2 \mathrm{~s}$ of continuous SWD activity (arrow, pellet). Presentation of the pellet consistently blocked SWDs. This effect is apparent in Figure $1 B$, where the percentages of SWD bursts of different duration were logged in an individual rat. Compared with no-reward periods, when burst lengths were 2-11 s (blue), during reward, burst lengths were nearly all $2 \mathrm{~s}$ (red). Examination of individual bursts indicated that some ended before the pellet presentation. However, most events appeared to be blocked by the reward itself, obscuring voluntary termination with exogenous (stimulus-based) blocking (Osterhagen et al., 2010).

Since we were interested in whether rats were conscious during SWD bursts, and could voluntarily terminate the bursts, we switched to a paradigm where bursts of any duration $\geq 3 \mathrm{~s}$ were rewarded, but the reward was delayed for $3 \mathrm{~s}$ following burst termination (Fig. $2 A$, pre, magenta). Thus, SWD bursts of any duration $\geq 3$ s were logged and SWD-blocking was avoided by only presenting the reward $3 \mathrm{~s}$ after the rat had stopped the burst. The delayed-reward paradigm also permitted us to examine when rats began checking the pellet tube in relation to the end of
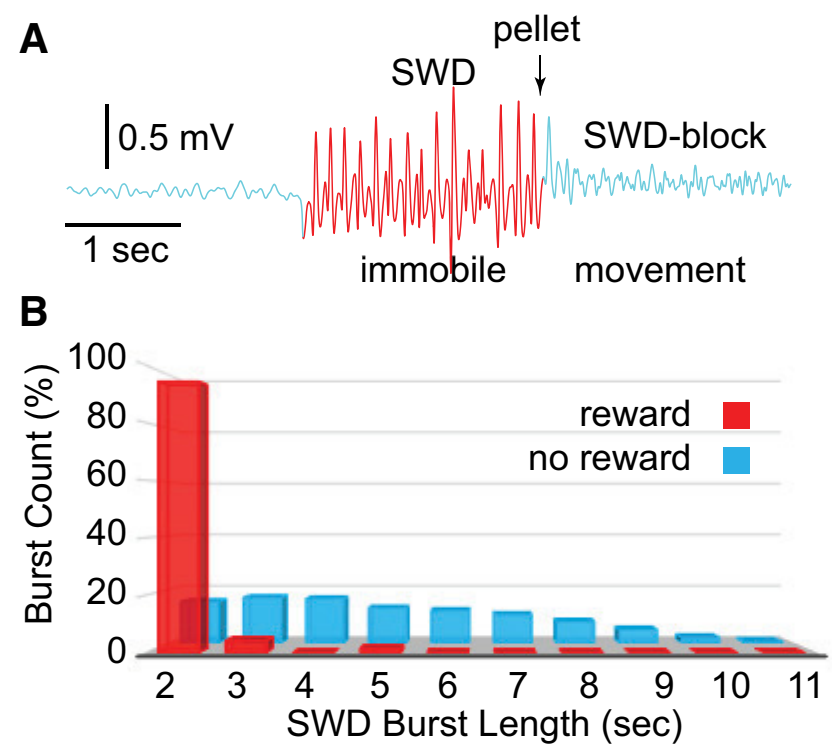

Figure 1. SWD blocking by pellet delivery in SD rats. $A$, An example of SWDs (red) that were automatically detected using an SVM trained separately for each animal. When the reward (pellet) was triggered after $2 \mathrm{~s}$ of SWDs, it regularly blocked the burst. $\boldsymbol{B}$, Counts of SWD bursts of lengths ranging from 2 to $11 \mathrm{~s}$ during reward (red) and no-reward (blue). Nearly all bursts were $2 \mathrm{~s}$ in length during reward due to blocking.

SWDs and presentation of the reward. Figure $2 A$ (blue trace) shows the output of an infrared-photodiode beam positioned at the pellet tube to detect the movement of the rat when checking for reward (indicated by interruption of the beam). In this example, the rat preemptively checked (Fig. $2 A$, first check) the tube after SWDs but before pellet delivery. Figure $2 B$ shows the timing of all first checks for reward (red; white noise cue on for $30 \mathrm{~min}$ ) and no-reward (blue; white noise cue off for $30 \mathrm{~min}$ ) conditions across all five SD rats. During reward, most first checks occurred within the first $3 \mathrm{~s}$ following SWD termination but before pellet delivery (Fig. $2 B$, arrow, pre). Because the rats preemptively checked for the expected reward after SWD completion but before delivery, the rats appeared to be cued by the end of SWDs and not just the sound of the pellet drop. During the no-reward condition, checking the pellet tube was diminished. Therefore, the behavior of checking for the reward was not a random activity independent of the SWDs; rather, it depended heavily on presentation of a reward after the SWDs.

It is well recognized, however, that SWDs in rats are associated with immobility, as they were in the present animals. SWDrelated immobility appears as an interruption of ongoing behavior, justifying the interpretation of SWD bursts as nonconvulsive seizures. If the rat's ongoing behavior was to randomly check the pellet tube for reward, and this behavior was interrupted by SWDs, post-SWD checking behavior could be due to a release from ictal immobility and not to anticipated reward for terminating the SWD burst. This explanation, however, did not fit our results. The average rate of checks across animals showed an eightfold increase during reward periods (Fig. $2 C$, red), indicating that random checking without reward (Fig. 2C, blue) was limited. During reward periods, $73 \%$ of the checks were performed in the $6 \mathrm{~s}$ post-SWD period (i.e., the $3 \mathrm{~s}$ before plus $3 \mathrm{~s}$ after pellet period). Thus, checking for rewards was clustered at the end of SWDs and not spread randomly throughout the interburst intervals (Fig. $2 B, C$, IBI), which were much longer in duration $(70 \pm 1.6 \mathrm{~s})$. Therefore, rats timed their checking for pellets in reference to the termination of SWDs. 


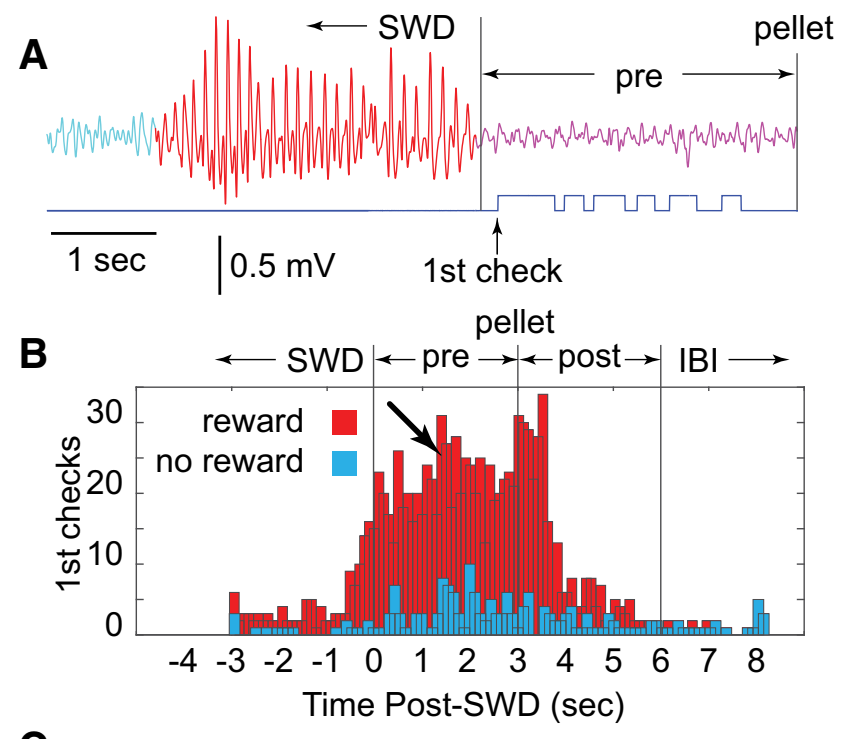

C

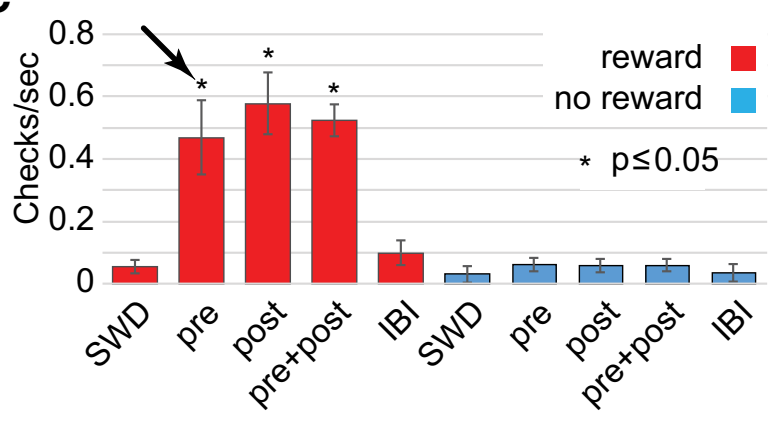

Figure 2. Delayed reward and preemptive checking in SD rats $(n=5)$. $\boldsymbol{A}$, With delayed reward, the SWD burst could be any length $\geq 3 \mathrm{~s}$ (in this example, the length was $3 \mathrm{~s}$ ) but had to be voluntarily terminated with $3 \mathrm{~s}$ of no SWD (magenta, pre) before the pellet was delivered, to avoid stimulus-induced blocking. Checking of the reward tube was detected by interruption of a photodiode (blue trace). $\boldsymbol{B}$, Histogram, computed across rats, of first checks (the initial interruption of the photodiode after SWD termination), during SWDs, following SWDs but before pellet delivery (pre, arrow), $3 \mathrm{~s}$ period following pellet delivery (post), and the beginning of the interburst interval (IBI). Most first checks were preemptive, before the pellet was delivered. C, The average rate of checking was higher during the prereward and postreward periods than during either SWD or interburst interval. Results shown as mean \pm SEM.

Preemptive checking for reward following SWDs suggested that rats were conscious during SWD bursts, which is not common for human absence seizures or CPSs. However, it was possible that the SWD bursts could be spontaneous seizures with maintenance of partial consciousness. We therefore examined whether rats could control the duration of SWDs: could the rats learn to shorten bursts during reward periods to obtain a pellet? Addressing this question, we concentrated on burst durations instead of the effect of reward condition on the total amount of SWDs, since the latter measure could be influenced by motor behaviors associated with reward delivery and consumption, rather than control over SWDs. Figure 3 presents the effect of reward on SWD burst durations. Without reward, SWD burst durations typically ranged between 3 and $10 \mathrm{~s}$ but many bursts extended past $10 \mathrm{~s}$. Figure $3 \mathrm{~A}$ (upper trace) shows an example of an unusually long $25 \mathrm{~s}$ SWD burst during no-reward. While the amplitude appeared to wax and wane, there was no significant change in waveform (i.e., spike-wave morphology) or frequency throughout and SVM scored it correctly as one prolonged burst. The temporal pattern of SWDs was markedly changed during the reward period (Fig. $3 A$, lower trace), with bursts concentrated in the range of $3-5 \mathrm{~s}$ and rarely $>9 \mathrm{~s}$. Figure $3 B$ displays a histogram reflecting changes in SWD burst durations during the first and second paired no-reward/reward $30 \mathrm{~min}$ periods (NR1/R1 and NR2/R2, respectively). Note here that, compared with Figure $1 B$, where $2 \mathrm{~s}$ SWD bursts dominated due to SWD-blocking at $2 \mathrm{~s}$, plots in Figure 3 begin at the shortest burst length of $3 \mathrm{~s}$ since a minimum length of $3 \mathrm{~s}$ was imposed during operant conditioning to define a burst. Two-way ANOVA revealed a significant interaction between reward condition and burst duration $\left(F_{(51,288)}=\right.$ $1.573, p=0.012)$. This was due to an overall shift in durations toward shorter $(3-5 \mathrm{~s})$ bursts during reward periods, compared with no-reward periods where burst durations were more evenly distributed and bursts $>10$ s were commonly observed (Fig. $3 B$, white arrows). Thus, SWDs in SD rats did not have the properties of spontaneous seizures; instead, SWDs appeared to be under operant control.

\section{Inbred WAG/Rij rats}

Because SWDs and associated immobility in healthy outbred SD rats differed from expectations for spontaneous seizures, we questioned how they differ from similar events in rats specifically inbred for SWDs and used as models of absence epilepsy. The essential difference in rats considered to be models of absence epilepsy could be one of diminished consciousness and voluntary control, not the presence or electrographic signature of the SWD itself. To answer this question, we used the same operant procedures with WAG/Rij rats, a strain specifically inbred for SWDs (Coenen and Van Luijtelaar, 2003). Our results supported the hypothesis of diminished consciousness and control in WAG/Rij rats. Even after $20 \mathrm{~d}$ of training, first checks for reward were most prevalent in the $3 \mathrm{~s}$ period following pellet delivery (Fig. 4A, post) as opposed to the preceding $3 \mathrm{~s}$ (Fig. $4 A$, pre, arrow). First checks were essentially absent during the no-reward condition. Similarly, the rate of preemptive checking did not significantly exceed those during SWDs with associated immobility (Fig. $4 B$ ) and only became significantly greater following delivery. The rats learned to check mainly after hearing the pellet drop and did not often anticipate delivery based on the moment of successful SWD termination. Furthermore, WAG/Rij rats showed no clear signs of voluntary control over SWD burst durations (Fig. 4C), and lacked significant interaction between reward condition and burst duration $\left(F_{(51,144)}=0.998, p=0.489\right)$.

Despite extensive training, WAG/Rij rats differed from our outbred SD controls in their consciousness during, and control over, SWD bursts, which was greatly diminished or absent. Together, these events reflected typical absence seizures in humans when genetically enhanced in the rat model. Yet, the question remained whether these animals could not, or would not, perform our SWD-related task. Perhaps the inbred strain was less motivated in general or at least less motivated by the sucrose pellet reward (possible anhedonia; Sarkisova and van Luijtelaar, 2011). So next, we food-deprived the rats the evening before each training session, and trained every other day to maintain proper nutrition and body weight. Surprisingly, food deprivation, and presumably increased motivation to perform the task, had a substantial effect on performance. Results for WAG/Rij rats became nearly indistinguishable from those of the nondeprived healthy outbred animals, with markedly increased preemptive checking of the pellet tube (Fig. 5A, arrow), significantly increased prereward check rates (Fig. $5 B$, arrow), and a return to significant interaction between reward condition and burst duration (Fig. $\left.5 C ; F_{(51,288)}=9.433, p<0.000\right)$. Similar to SWD burst lengths in healthy SD rats, SWD burst lengths in WAG/Rij rats appeared 


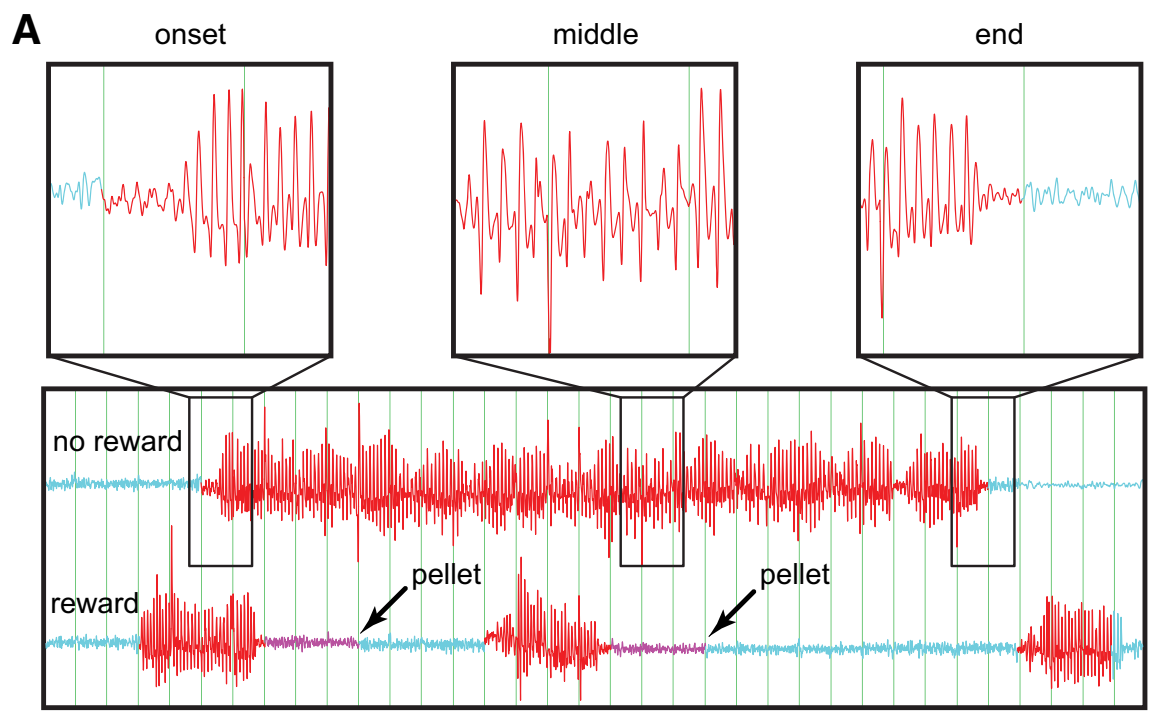

B

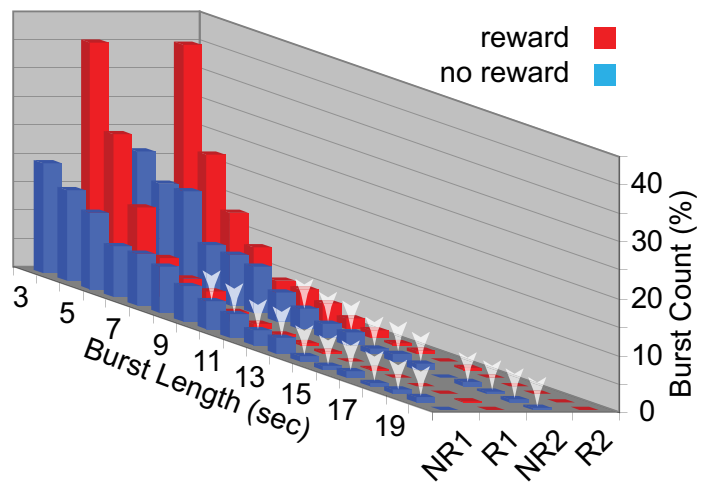

Figure 3. The effect of reward on SWD burst lengths in SD rats $(n=5)$. $A$, Even during particularly long SWD bursts, such as the 25 epoch example shown here during no reward (upper trace), the frequency and waveform remained sufficiently constant to be counted as a single burst (red trace) by SVM. In contrast, during reward (lower trace), SWDs were typically terminated after only several seconds, followed by $3 \mathrm{~s}$ (magenta) before reward presentation (arrows). B, During the first (NR1) and second (NR2) no-reward periods (white noise cue off), burst lengths (blue) were widely distributed, ranged from 3 to $20 \mathrm{~s}$. Bursts $>10 \mathrm{~s}$ are marked with white arrows. During the first and second reward periods (R1 and R2, respectively; white noise cue on), SWD burst lengths (red) were shortened to mainly $3-5 \mathrm{~s}$.

under voluntary control, albeit a result requiring greater motivation to achieve.

\section{Outbred LE rats}

The similarity between SD and WAG/Rij rats suggested that SWDs in inbred WAG/Rij rats were not easily distinguished from healthy outbred SD controls. However, there remained an alternative possibility, that the presence of SWDs in our outbred SD rats was strain and/or colony specific and not representative of healthy outbred animals. Genetic drift over multiple generations of SD rats could have inadvertently favored SWDs in this strain. To examine this possibility, we conducted a final experiment to test the generality of our findings by applying operant training to LE rats, another outbred strain commonly used in behavioral neuroscience experiments. To avoid confounds of motivation encountered with $W A G / R i j$ rats, all LE rats were trained with food deprivation from the outset. Results for these rats were similar to those obtained in SD and WAG/Rij rats (Fig. 6). As shown in Figure 6A, LE rats detected the termination of their SWD bursts and preemptively checked the pellet tube in advance of reward presentation (Fig. 6A, arrow). Like SD rats, LE rats demonstrated an increase in first checks following the pellet drop, presumably cued by the sound. Prereward checking in LE rats was also high during the noreward condition, indicating dependence on cues based on SWD burst completion alone, independent of reward presentation. Rats tended to do the most checking at the end of SWDs, and especially during the $3 \mathrm{~s}$ preceding and following pellet drop. Checking was less common during the period of SWD-associated immobility and the long interburst interval (Fig. 6B). As in the SD and food-deprived WAG/Rij rats, there was a significant interaction between reward condition and burst duration (Fig. $\left.6 C ; F_{(51,288)}=4.6, p<0.000\right)$. Voluntary shortening of SWD burst length was possible in LE rats as it was in our other groups, suggesting the phenomenon may be general and not strain or colony specific.

\section{Similarity of SWDs between strains}

In addition to finding behavioral similarities between the three rat strains, we wished to determine whether SWDs across strains were a comparable phenomenon. To this end, we examined the sensitivity of SWDs to ethosuximide, and also performed power spectral analysis to compare strain-related signatures of SWD frequency.

Ethosuximide, a T-type calcium channel antagonist that is a common treatment for absence seizures in humans and that has been demonstrated to block SWDs in both inbred (Coenen and Van Luijtelaar, 2003) and outbred (Pearce et al., 2014) rats, was used to determine whether the pharmacological sensitivity of SWDs in our rats differed in some way from that observed in other experiments, and could therefore represent a unique phenomenon in one or more of the strains used here. As shown in Figure 7, ethosuximide profoundly suppressed SWD rates of all strains in the $1 \mathrm{~h}$ recording period following injection (Ethosux) compared with recording periods $24 \mathrm{~h}$ before (Pre) and after (Post) injection, similar to previous reports for SD rats (Pearce et al., 2014). Figure 7 also shows an unexpected result from this study. The baseline rates of SWDs (Pre and Post) in WAG/Rij rats did not significantly differ from those of SD rats, and were significantly less than those of the LE rats $(p<0.01)$. Together, these results suggested the SWDs were similar in pharmacosensitivity and, thus, similar phenomena across strains.

This conclusion was reinforced by power spectral analysis (Fig. 8). The spike-wave morphology of SWDs in all strains resulted in a signal that was quasiperiodic, having unique spectra with high values at a fixed fundamental frequency (e.g., $8-10 \mathrm{~Hz}$ ) and at whole multiples (harmonics) of this fundamental frequency (e.g., 16-20 and 24-30 Hz; Fig. 8A, B; arrows). To com- 
A

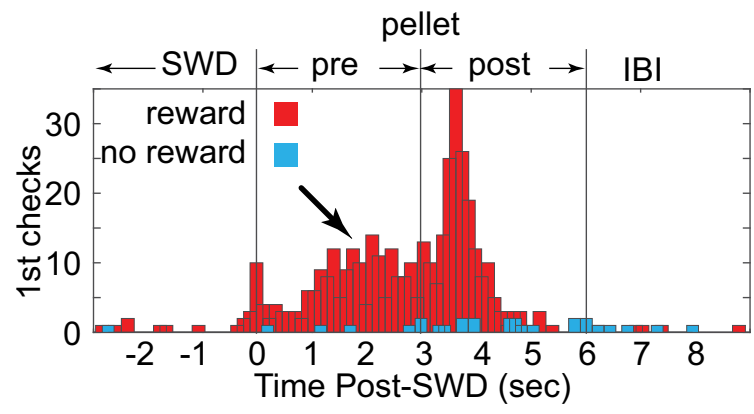

B

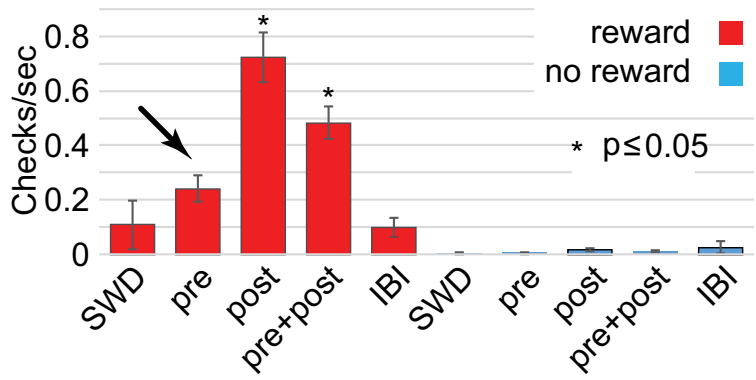

C

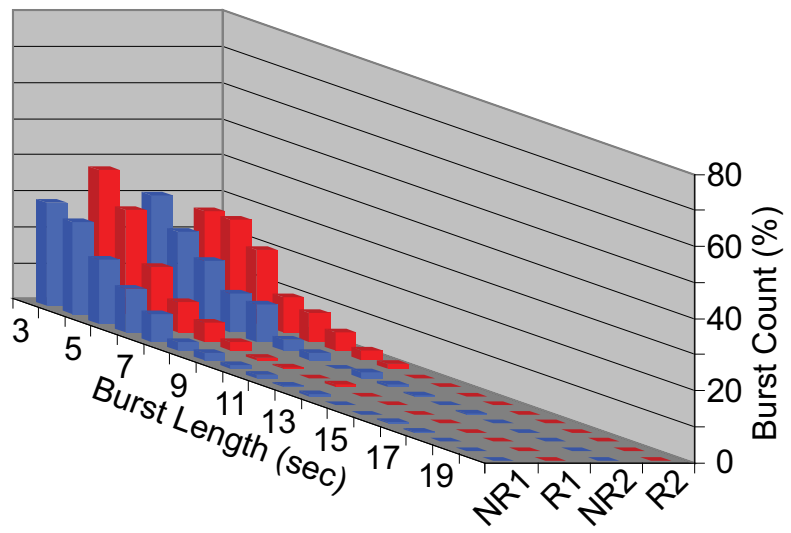

Figure 4. SWD bursts and reward in WAG/Rij rats $(n=5)$ without food deprivation. $A$, In contrast to SD rats, WAG/Rij rats showed less preemptive checking of the reward tube (arrow) during reward periods (red) with most first checks following pellet delivery. Checks during no-reward periods (blue) were nearly absent. $\boldsymbol{B}$, This was also reflected in the overall rates of checking, which only significantly increased from the SWD and interburst interval (IBI) periods following reward. Again, rates of checking were greatly diminished during the no-reward periods. C, Similarly, burst durations appeared to be uninfluenced by reward.

pare the spectra between rat strains, they were averaged within each strain (Fig. 8B). The spectra were highly correlated between all strains with correlation coefficients ranging from 0.86 to 0.96 ( $p<0.000$ in all comparisons).

\section{Discussion}

Our results demonstrate the following: (1) SWD and associated immobility events in inbred WAG/Rij and outbred SD and LE rats are indistinguishable on all measured parameters, including suppression by ethosuximide, and are therefore very similar if not identical phenomena; (2) rats in all groups can detect SWDs, begin preemptively checking for rewards, and shorten their duration to obtain reward, indicating maintenance of at least partial consciousness and the capacity of voluntary control; and (3) rats in all groups alter their operantly conditioned behavior in relation to the presence or absence of a reward-period cue (white noise), suggesting maintenance of not just consciousness but cognitive function during SWD and associated immobility.
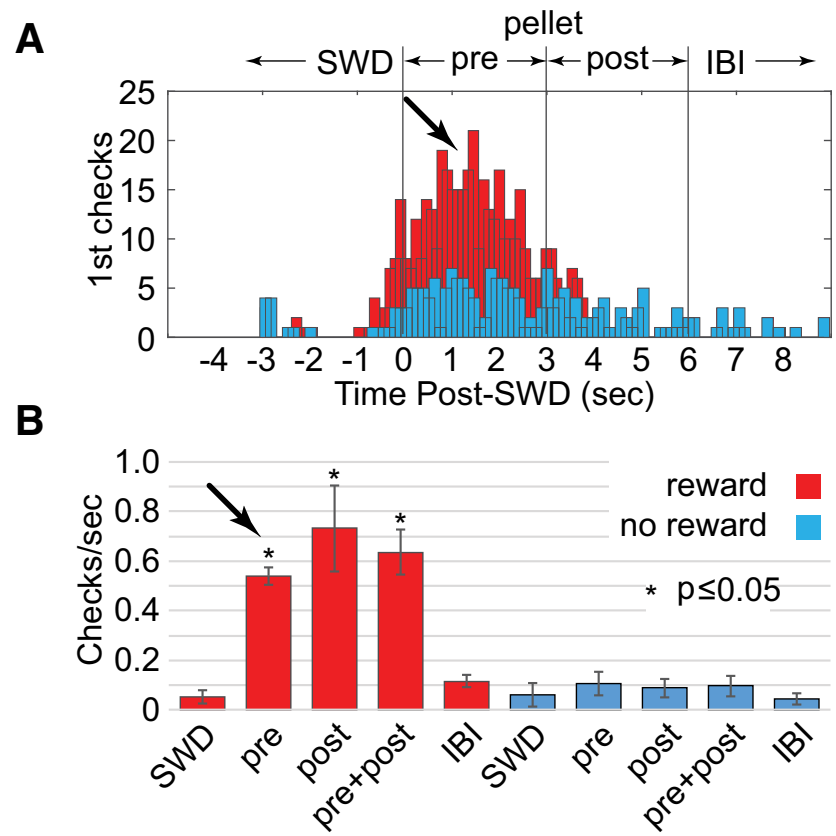

C

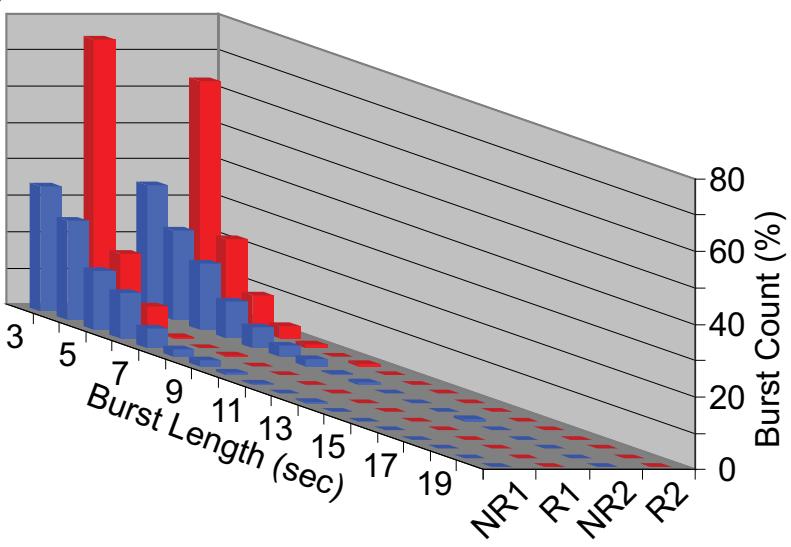

Figure 5. SWD bursts and reward in WAG/Rij rats $(n=5)$ with food deprivation. The results shown in Figure 4 were apparently due to lower motivation in WAG/Rij rats to perform the task. $A-C$, Following food deprivation, preemptive checking $(\boldsymbol{A})$, overall check rates $(\boldsymbol{B})$, and rewardbased decreases in SWD burst duration $(\boldsymbol{C})$ resembled those of nondeprived SD rats.

\section{SWDs and associated immobility as a model of CPSs}

Several laboratories have reported SWDs and associated immobility in SD rats as evidence for CPSs in rat models of acquired epilepsy, such as those following FPI in adults (D'Ambrosio et al., 2004), neonatal hypoxia-induced seizures (Rakhade et al., 2011), and hyperthermia-induced seizures in immature rats (Dubé et al., 2006). These publications have reported that SWD-like events only occur after the respective brain insult and that they become more prominent with increased time after the brain insult. Therefore, it has been proposed that these events are analogous to human focal CPSs (D'Ambrosio et al., 2009). The notion that SWDs reflect CPSs has been challenged by evidence of equal prevalence in age-matched uninjured rats (Pearce et al., 2014; Rodgers et al., 2015). One recent report distinguished only SWD bursts lasting $>10 \mathrm{~s}$ as PTE, because bursts of this duration were not observed in uninjured controls (Reid et al., 2016). The present data (and see Rodgers et al., 2015), however, indicate that in healthy outbred rats with no insult, SWDs $>10 \mathrm{~s}$ are common though less frequent than shorter bursts (Figs. $3 A, B, 6 C$ ), particularly in older rats ( $>6$ months; Rodgers et al., 2015) with or 
A

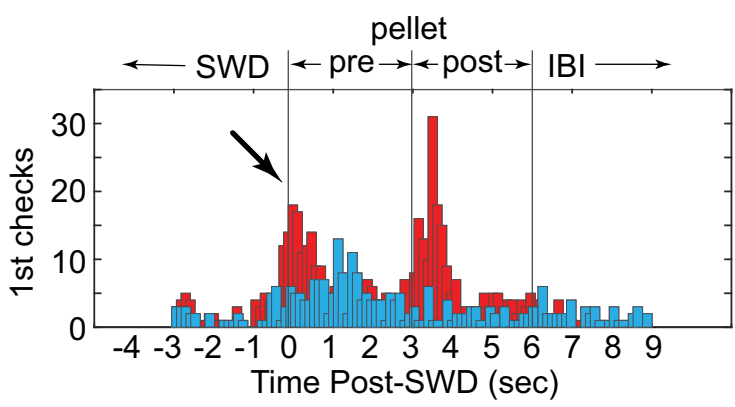

B

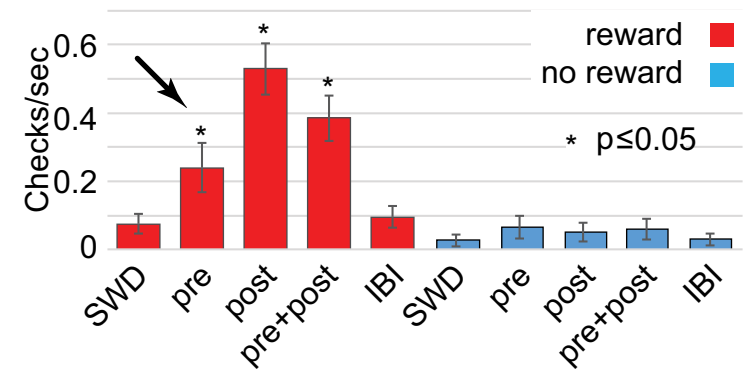

C

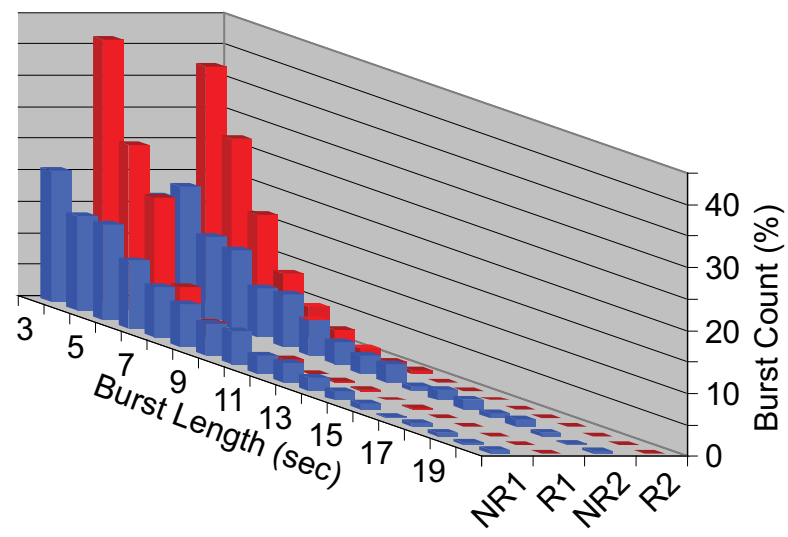

Figure 6. SWD bursts and reward in LE rats $(n=5)$ with food deprivation. A, Preemptive first checks were apparent in the first seconds following SWD bursts (arrow) but before reward. There was also an increase in first checks following reward, presumably due to the sound of pellet drop. $\boldsymbol{B}$, Preemptive checking rates (pre) were significantly greater than those seen during either SWDs or during the interburst interval, as were rates postreward. C, SWD durations were significantly shorter during the two reward periods (red) compared with the two noreward periods (blue).

without injury. While it is therefore unlikely that SWDs are related to brain injury and PTE (Kelly et al., 2006), the present study provides an objective method for determining whether such events following brain insults differ from controls and are insensitive to operant control, as would be expected for CPSs if there were no learning deficit due to injury.

\section{SWDs and associated immobility as a model of human absence epilepsy}

Human absence seizures have classically been defined by obvious impairment of consciousness (i.e., absence), with a concomitant lack of awareness and responsiveness synchronous with cessation of activities (i.e., behavioral interruption) that occur spontaneously (i.e., seizure; Hughes, 2009; Berg et al., 2010). An absence seizure is sudden in onset and offset, and typically has a duration in the range of 3 to $20 \mathrm{~s}$ with regular $3 \mathrm{~Hz}$ SWDs in the scalp EEG (Stefan and Snead, 2008). Based on their genetic predisposition
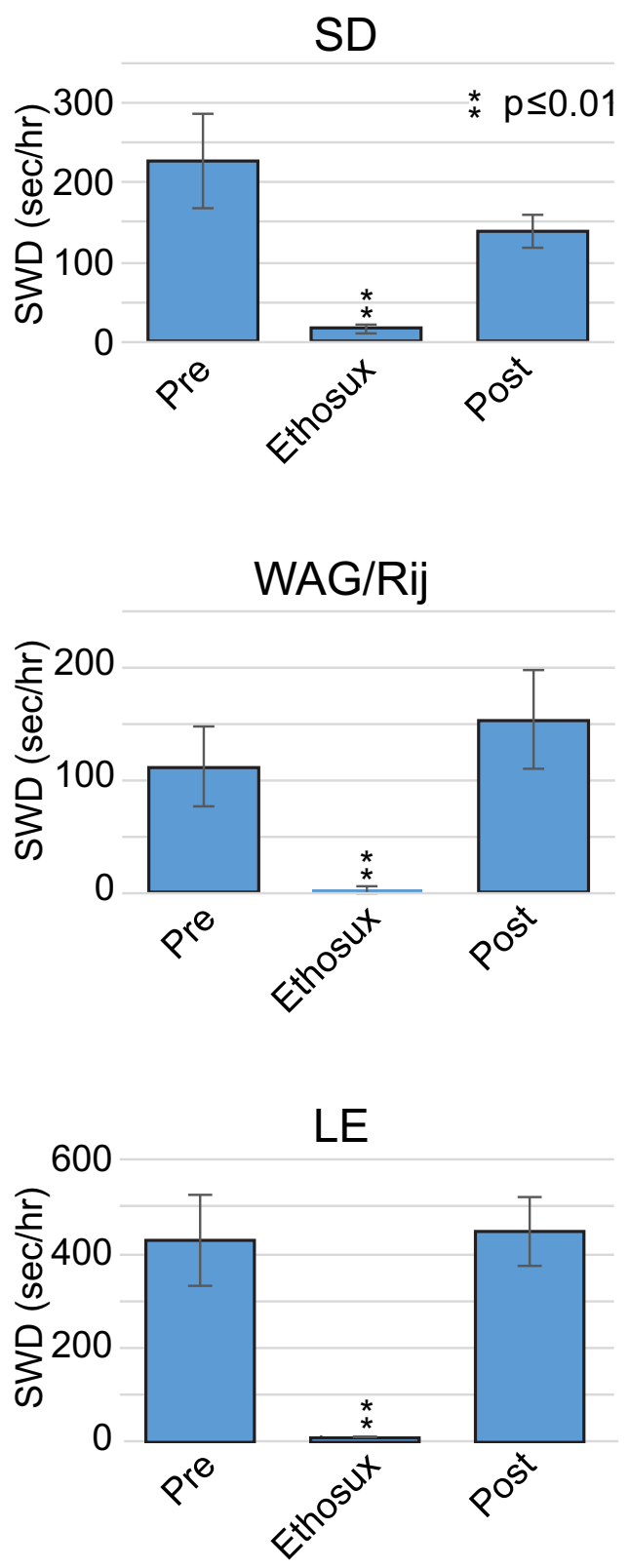

Figure 7. The effect of ethosuximide on SWD burst rates in SD, WAG/Rij, and LE rats ( $n=$ 5/group). Rates are shown for the $1 \mathrm{~h}$ recording block $24 \mathrm{~h}$ before injection (Pre) compared with similar recordings in blocks following injection (Ethosux) and after $24 \mathrm{~h}$ recovery (Post).

and ictal manifestations, WAG/Rij rats and Genetic Absence Epilepsy Rats from Strasbourg (GAERS) - selectively inbred for SWDs for what is now over 100 generations-have face validity as models of the human condition. They also have predictive validity in that human antiabsence drugs (e.g., ethosuximide, as shown here) suppress SWDs in rats (Marescaux et al., 1992). However, our results indicate that the SWDs of WAG/Rij rats do not model this classic definition of human absence epilepsy (except perhaps in unmotivated rats), since consciousness seems to be maintained during SWDs, permitting voluntary control. Other laboratories have also shown a reduction of SWDs in WAG/Rij rats during operant conditioning (Van Luijtelaar et al., 1991; Osterhagen et al., 2010), although it was unclear in these studies if this reduction was voluntary or due to reward/arousalinduced blocking, since rewards were presented during SWDs with no delay. 


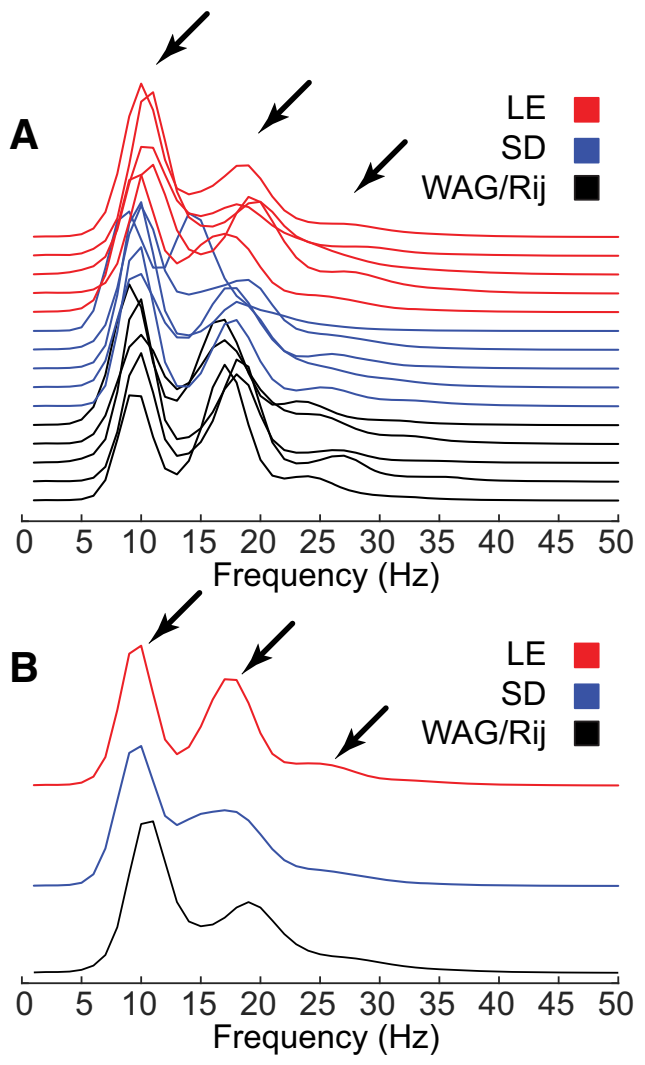

Figure 8. Spectral analysis of SWDs in SD, WAG/Rij, and LE rats ( $n=5 /$ group). $\boldsymbol{A}$, Power spectra were similar in all strains, conforming to that expected for a quasiperiodic signal with peaks at a fundamental frequency and harmonic multiples of that frequency (arrows). $\boldsymbol{B}$, Averaged power spectra within each rat strain.

Consciousness can be partially maintained during mild human absence seizures, and the extent of impairment can vary between seizures and subjects (Blumenfeld, 2005, 2012). WAG/ $R i j$ rats model these milder absence seizures with partial impairment of consciousness. This conclusion, if true, has two important implications. First, it suggests that milder absence seizures in humans might be brought under operant control. It stands to reason that during absence seizures where consciousness is partially maintained, operantly conditioned arousal (Van Luijtelaar et al., 1991) upon recognition of the seizure might significantly shorten its duration as demonstrated here in rats. To our knowledge, this possibility has not been tested in humans. Second, this conclusion would suggest that rats used in behavioral and preclinical experiments could suffer from mild absence epilepsy. SWD and associated immobility events in WAG/Rij rats are indistinguishable from those in the outbred strains in all measurable parameters, including pharmacosensitivity, waveform morphology, frequency, duration, sensory blocking, and immobility, as well as sensitivity to operant control. Additionally, at least in our sample of adult outbred animals, SWDs were as prevalent in SD rats, and more prevalent LE rats, than in the WAG/Rij rats. If healthy outbred rats are prone to numerous spontaneous partial lapses of both consciousness and mobility, these results indicate a critical need for chronic video/EEG monitoring of experimental and control animals to detect possible confounds.

\section{SWDs and associated immobility as voluntary behavior} It is also possible that the occurrence of SWD bursts with associated immobility at least in healthy outbred rats reflects part of their behavioral repertoire and not epilepsy. Our observations of SWDs in outbred rats are common to several previous studies of SD (Aldinio et al., 1985; Kaplan, 1985; Kleinlogel, 1985; Buzsaki et al., 1990b; Kelly et al., 2001; Kharlamov et al., 2003; Pearce et al., 2014) and LE (Semba et al., 1980; Kaplan, 1985; Wiest and Nicolelis, 2003; Shaw, 2004) rats, as well as in outbred Wistar (Robinson and Gilmore, 1980; Vergnes et al., 1982; Kaplan, 1985) and hooded (Vanderwolf, 1975) rats. This electrographic pattern has been variously termed polyspiking activity, sensorimotor rhythm, high-voltage spindles, $\alpha$ (mu) rhythm, paroxysmal electroclinical patterns, and high-voltage rhythmic spike discharge, as well as SWDs. In addition to remarkably similar waveform morphologies, SWD-like events in these reports consistently range from 7 to $12 \mathrm{~Hz}$ with amplitudes of $>200 \mu \mathrm{V}$. SWDs are regularly noted to start and stop abruptly and are associated with inactivity and behavioral arrest, vibrissa tremor, and facial movements. Observation of similar SWD-like events across various studies and strains of adult outbred rats suggests ubiquity lacking a distinct genetic component and may indicate their unreliability as a signature of epileptic seizures.

It has been observed here and previously (Vergnes et al., 1982; Wiest and Nicolelis, 2003; Pearce et al., 2014; Rodgers et al., 2015) that SWDs may be blocked by mild sensory stimuli. Rats can also sense and respond to stimuli during SWDs (Wiest and Nicolelis, 2003; Chipaux et al., 2013). However, the ability to respond to sensory stimulation during SWDs does not demonstrate that they are not seizures, since human patients have been reported to show responsiveness of sensory cortex during seizures (Chipaux et al., 2013). What our data indicate is the following: (1) rats are at least partially conscious during SWD events; (2) they remember and combine this information with a contextual cue (e.g., presence or absence of continuous white noise signaling reward/noreward condition) to correctly perform an operant task; and (3) during reward conditions, rats terminate SWDs early and preemptively seek an expected reward. It remains to be determined whether an analogous level of consciousness and cognitive/behavioral function is preserved during mild absence seizures in humans. Further studies are also needed to directly determine whether SWD and associated immobility events in adult rats interfere with performance in behavioral experiments or whether their impact is mitigated by the level of retained cognitive function and/or voluntary control demonstrated here.

\section{References}

Aldinio C, Aporti F, Calderini G, Mazzari S, Zanotti A, Toffano G (1985) Experimental models of aging and quinolinic acid. Methods Find Exp Clin Pharmacol 7:563-568. Medline

Berg AT, Berkovic SF, Brodie MJ, Buchhalter J, Cross JH, van Emde Boas W, Engel J, French J, Glauser TA, Mathern GW, Moshé SL, Nordli D, Plouin P, Scheffer IE (2010) Revised terminology and concepts for organization of seizures and epilepsies: Report of the ILAE Commission on Classification and Terminology, 2005-2009. Epilepsia 51:676-685. CrossRef Medline

Blumenfeld H (2005) Consciousness and epilepsy: why are patients with absence seizures absent? Prog Brain Res 150:271-286. CrossRef Medline

Blumenfeld H (2012) Impaired consciousness in epilepsy. Lancet Neurol 11:814-826. CrossRef Medline

Buzsáki G, Laszlovszky I, Lajtha A, Vadasz C (1990a) Spike-and-wave neocortical patterns in rats: genetic and aminergic control. Neuroscience 38:323-333. CrossRef

Buzsáki G, Smith A, Berger S, Fisher LJ, Gage FH (1990b) Petit mal epilepsy and parkinsonian tremor: hypothesis of a common pacemaker. Neuroscience 36:1-14. CrossRef Medline

Chipaux M, Vercueil L, Kaminska A, Mahon S, Charpier S (2013) Persistence of cortical sensory processing during absence seizures in human and 
an animal model: evidence from EEG and intracellular recordings. PLoS One 8:e58180. CrossRef Medline

Coenen AM, Van Luijtelaar EL (2003) Genetic animal models for absence epilepsy: a review of the WAG/Rij strain of rats. Behav Genet 33:635-655. CrossRef Medline

D’Ambrosio R, Fairbanks JP, Fender JS, Born DE, Doyle DL, Miller JW (2004) Post-traumatic epilepsy following fluid percussion injury in the rat. Brain 127:304-314. CrossRef Medline

D’Ambrosio R, Hakimian S, Stewart T, Verley DR, Fender JS, Eastman CL, Sheerin AH, Gupta P, Diaz-Arrastia R, Ojemann J, Miller JW (2009) Functional definition of seizure provides new insight into post-traumatic epileptogenesis. Brain 132:2805-2821. CrossRef Medline

D'Ambrosio R, Miller JW (2010) What is an epileptic seizure? Unifying definitions in clinical practice and animal research to develop novel treatments. Epilepsy Curr 10:61-66. CrossRef Medline

Dubé C, Richichi C, Bender RA, Chung G, Litt B, Baram TZ (2006) Temporal lobe epilepsy after experimental prolonged febrile seizures: prospective analysis. Brain 129:911-922. CrossRef Medline

Dudek FE, Bertram EH (2010) Counterpoint to "What Is an Epileptic Seizure?” By D’Ambrosio and Miller. Epilepsy Curr 10:91-94. CrossRef Medline

Hughes JR (2009) Absence seizures: a review of recent reports with new concepts. Epilepsy Behav 15:404-412. CrossRef Medline

Kaplan BJ (1985) The epileptic nature of rodent electrocortical polyspiking is still unproven. Exp Neurol 88:425-436. CrossRef Medline

Kelly KM (2004) Spike-wave discharges: absence or not, a common finding in common laboratory rats. Epilepsy Curr 4:176-177. CrossRef Medline

Kelly KM, Kharlamov A, Hentosz TM, Kharlamova EA, Williamson JM, Bertram EH 3rd, Kapur J, Armstrong DM (2001) Photothrombotic brain infarction results in seizure activity in aging Fischer 344 and Sprague Dawley rats. Epilepsy Res 47:189-203. CrossRef Medline

Kelly KM, Jukkola PI, Kharlamov EA, Downey KL, Mcbride JW, Strong R, Aronowski J (2006) Long-term video-EEG recordings following transient unilateral middle cerebral and common carotid artery occlusion in Long-Evans rats. Exp Neurol 201:495-506. CrossRef Medline

Kharlamov EA, Jukkola PI, Schmitt KL, Kelly KM (2003) Electrobehavioral characteristics of epileptic rats following photothrombotic brain infarction. Epilepsy Res 56:185-203. CrossRef Medline

Kleinlogel H (1985) Spontaneous EEG paroxysms in the rat: effects of psychotropic and alpha-adrenergic agents. Neuropsychobiology 13:206213. CrossRef Medline

Marescaux C, Vergnes M, Depaulis A (1992) Genetic absence epilepsy in rats from Strasbourg-a review. J Neural Transm Suppl 35:37-69. Medline

Nandan M, Talathi SS, Myers S, Ditto WL, Khargonekar PP, Carney PR (2010) Support vector machines for seizure detection in an animal model of chronic epilepsy. J Neural Eng 7:036001. CrossRef Medline

Orrù G, Pettersson-Yeo W, Marquand AF, Sartori G, Mechelli A (2012) Using support vector machine to identify imaging biomarkers of neuro- logical and psychiatric disease: a critical review. Neurosci Biobehav Rev 36:1140-1152. CrossRef Medline

Osterhagen L, Breteler M, van Luijtelaar G (2010) Does arousal interfere with operant conditioning of spike-wave discharges in genetic epileptic rats? Epilepsy Res 90:75-82. CrossRef Medline

Pearce PS, Friedman D, Lafrancois JJ, Iyengar SS, Fenton AA, Maclusky NJ, Scharfman HE (2014) Spike-wave discharges in adult Sprague-Dawley rats and their implications for animal models of temporal lobe epilepsy. Epilepsy Behav 32:121-131. CrossRef Medline

Rakhade SN, Klein PM, Huynh T, Hilario-Gomez C, Kosaras B, Rotenberg A, Jensen FE (2011) Development of later life spontaneous seizures in a rodent model of hypoxia induced neonatal seizures. Epilepsia 52:753765. CrossRef Medline

Reid AY, Bragin A, Giza CC, Staba RJ, Engel J Jr (2016) The progression of electrophysiologic abnormalities during epileptogenesis after experimental traumatic brain injury. Epilepsia 57:1558-1567. CrossRef Medline

Robinson PF, Gilmore SA (1980) Spontaneous generalized spike-wave discharges in the electrocorticograms of albino rats. Brain Res 201:452-458. CrossRef Medline

Rodgers KM, Dudek FE, Barth DS (2015) Progressive, seizure-like, spike-wave discharges are common in both injured and uninjured Sprague-Dawley rats: implications for the fluid percussion injury model of post-traumatic epilepsy. J Neurosci 35:9194-9204. CrossRef Medline

Sarkisova K, van Luijtelaar G (2011) The WAG/Rij strain: a genetic animal model of absence epilepsy with comorbidity of depression. Prog Neuropsychopharmacol Biol Psychiatry 35:854-876. CrossRef Medline

Semba K, Szechtman H, Komisaruk BR (1980) Synchrony among rhythmical facial tremor, neocortical "alpha" waves, and thalamic non-sensory neuronal bursts in intact awake rats. Brain Res 195:281-298. CrossRef Medline

Shaw FZ (2004) Is spontaneous high-voltage rhythmic spike discharge in Long Evans rats an absence-like seizure activity? J Neurophysiol 91: 63-77. Medline

Stefan H, Snead OC, III (2008) Typical and atypical absence seizures, myoclonic absences, and eyelid myoclonia. In: Epilepsy: a comprehansive textbook, 2nd ed, pp 537-584. New York: Lippincott Williams \& Wilkins.

Van Luijtelaar EL, Van der Werf SJ, Vossen JM, Coenen AM (1991) Arousal, performance and absence seizures in rats. Electroencephalogr Clin Neurophysiol 79:430-434. CrossRef Medline

Vanderwolf CH (1975) Neocortical and hippocampal activation relation to behavior: effects of atropine, eserine, phenothiazines, and amphetamine. J Comp Physiol Psychol 88:300-323. CrossRef Medline

Vergnes M, Marescaux C, Micheletti G, Reis J, Depaulis A, Rumbach L, Warter JM (1982) Spontaneous paroxysmal electroclinical patterns in rat: a model of generalized non-convulsive epilepsy. Neurosci Lett 33:97101. CrossRef Medline

Wiest MC, Nicolelis MA (2003) Behavioral detection of tactile stimuli during 7-12 Hz cortical oscillations in awake rats. Nat Neurosci 6:913-914. CrossRef Medline 